\title{
Design of amplitude and phase modulated pulse trains with good auttocorrelation properties for radar communications
}

\author{
S.J Rosli ${ }^{1}$, H.A. Rahim ${ }^{2}$, K.N. Abdul Rani ${ }^{3}$ \\ ${ }^{1,2}$ School of Computer and Engineering, Universiti Malaysia Perlis, Malaysia \\ ${ }^{3}$ Department of Electronic Engineering Technology, Universiti Malaysia Perlis, Malaysia
}

\begin{tabular}{l}
\hline \hline Article Info \\
\hline Article history: \\
Received Oct 12, 2018 \\
Revised Dec 9, 2018 \\
Accepted Dec 21, 2018 \\
\hline
\end{tabular}

Keywords:

Autocorrelation Function Energy Ratio

Radar Communication

Sidelobe Energy

\begin{abstract}
Development of technique for synthesizing multilevel sequences with good correlation properties is very useful for several radar applications where as a set of phase and amplitude coded sequences will be synthesized directly for compression technique. In reality, the signal processing also been significant to transmit or store signals, to enhance desired signal component and to extract useful information carried by signals. Consequently, this paper describes affectively methods to generating the finite length multilevel sequence of any length that have low side lobe energy (SLE) and improved energy ratio (ER) in their autocorrelation function (ACF). Testing for the stability and the analyzing of systems zero pattern using z-transform for the generating sequence indicates a possible position of roots in the radius of circle lies. This is illustrated by application of 13-element Huffman code as a starting sequence, this technique more low complexity and compatible compared inverse filtering technique.
\end{abstract}

Copyright () 2019 Institute of Advanced Engineering and Science. All rights reserved.

\section{Corresponding Author:}

Hasliza Rahim,

School of Computer and Communication Engineering,

University Malaysia Perlis,

Tingkat 1, Kampus Tetap Pauh Putra, 02600 Arau, Perlis, Malaysia.

Email: haslizarahim@unimap.edu.my

\section{INTRODUCTION}

Most modern high performance radars use travelling-wave tube amplifiers to obtain coherent transmission [1]. As pointed out previously, these tubes work more efficiently under constant amplitude conditions. Moreover, good amplitude modulation (AM) is difficult (and very expensive) to achieve with these devices. Therefore, only purely phase modulated pulse trains have been considered so far. However, with the emergence of solid state microwave sources, efforts are being made to replace the relatively large and expensive vacuum devices by low power solid state elements and the waveguide elements by planar circuits. With these new components the size and cost are reduced and a number of commercial applications become feasible [2].

The contribution of solid-state devices has also been significant in areas where performance was previously inadequate. For example, it is much easier to use any form of modulation with solid state components [3]. Although AM is not an efficient method to provide the large time-band width required for good radar performance, [4,5] it does provide an excellent means of improving the resolution capability. Consequently, this chapter treats the problem of finding ER amplitude and phase modulated (a.m and p.m) or multilevel sequence. 


\section{CHOICE OF STARTING SEQUENCE}

The choice of the starting sequence is an important process to determine the best sequence of random variables, describing an outcome following the deterministic pattern and probability distributions. These constructs are extremely useful in probability theory.

\subsection{Choice at Random}

Random sequence with optional properties that use property such as integer or length value are able to specify in any methods and any order to get the possibilities sequence intended for serving as a starting sequence.

The design of a Huffman sequence in general requires the choice of the code length $(\mathrm{N}+1)$, the circle radius $X$ and the zero pattern for which the magnitude sequence $|a(n)|$ is most uniformly distributed. However, it remains as an unsolved problem. The direct evaluation of all $2 \mathrm{~N}$ possible root pattern for a given radius $\mathrm{X}$ and $\mathrm{N}$ is not feasible if $\mathrm{N}$ is larger $(\mathrm{N}>20)$. It remains invariant even after account is taken of the zero patterns formed from others by rotation in the Z-plane through an angle $\emptyset$ or by other transformations. A general trial of the error procedure was done to chooe a root pattern randomly, to compute the sequence for a succession of values for radius $\mathrm{X}[6,7]$.

This method focuses more into this problem including the stationary phase principle applicable for an arbitrary length sequence. Zero pattern is one of the random solution on this method. In fact, Huffman, 1962 suggested that in order to maximize the ER, the roots should be chosen in a random fashion with approximately half the zeros on each circle. He further concluded that the optimum ER should be proportional to $(\mathrm{N}+1)-1 / 2$. Generally known that the correlation properties are used to judge the random sequence whereby the sequence is uncorrelated with itself, i.e. random, if its ACF has uniformly low side lobes. Although various methods as described above is acceptable for designing Huffman codes with moderately large length $(\mathrm{N}=100)$, the major difficulty arises for longer sequences because of the difficulty of the computational nature due to factorizing polynomials of degrees which are larger than 100 . The following sections describe some useful methods for designing the multilevel sequences [8].

\subsection{Designing Multilevel Sequences using $m$ integer and $N$ length}

To generate the multilevel sequences the length and some integer is considered. The impulseequivalent sequence is a finite sequence of complex numbers whose autocorrelation is zero $\mp \mathrm{N}$ [9]. The zero patterns in Figure 2.3 had been discussed in Chapter 2. The zeros of $\mp \mathrm{N}$ lies on 2 circles is an impulse equivalent which is a complex number of finite sequences.

a) Select $m$ with any integer $(m=1,2,3 \ldots)$ and any length of ' $N$ '.

b) X-X-1 should be an integer coefficient and $\frac{N}{2}$ is an odd integer and therefore choose $\mathrm{X}$ to be the positive root of $\mathrm{X}$ $\mathrm{X}-1$

$$
\begin{aligned}
& a^{2}-b X-c=0 \\
& X=\frac{-b \pm \sqrt{b^{2}-4 a c}}{2 a}
\end{aligned}
$$

Equation 1 is a quadratic equation for: Using 'quadratics coefficient', applying this formula (Equation 3).

$$
X^{2}-m X-1=0
$$

a) Generated $X^{N / 2}-X^{-N / 2}$.

b) Using 4 conditions (Equation 4,5,6 and 7) for generated integer values following length $\mathrm{N}$ :

For length 1 until 3

$$
H(1)=1, H(2)=2 m, H(3)=2 M^{2}
$$

For length 4 until $N+1$

The centre element of $\square=\left(\frac{N}{2}\right)+1$

$$
H(k)=m H\left(\frac{N}{2}\right)+H\left(\frac{N}{2}-1\right)-H(1) *\left(X \frac{N}{2}-X \frac{-N}{2}\right)
$$


For length $N$ is $K>\frac{N}{2}+1$

$$
H(L-k N)=-[H(K n+1)](-1)^{k N}
$$

If else of 3 conditions above

$$
H(k)=m H(k-1)+H(k-2)
$$

Table 1 shows is the set of sequences with 13-length of sequences and $m=1,2,3$, and 4 designing by multilevel sequences generated by $\mathrm{m}$ integer and $\mathrm{N}$ length produces $\mathrm{m}(\mathrm{x})$ sequences.

Table 1. Sequences Generated by Integer Values of $\mathrm{m}$

\begin{tabular}{cccccc}
\hline $\mathrm{L}$ & $\mathrm{m}$ & Sequence & S.L.E & E.R & P.S.E \\
\hline 13 & 1 & {$[1224610-1.8910-64-22-1]$} & 1.1723 & 3.2557 & 0.6880 \\
& 2 & {$[148204811682.01116-4820-84-1]$} & 0.9723 & 2.9138 & 0.4856 \\
& 3 & {$[161860198654862654-19860-186-1]$} & 1.0911 & 2.2674 & 0.6692 \\
& 4 & {$[1832136576244045582440-576136-328-1]$} & 0.9703 & 1.6070 & 0.6663 \\
\hline
\end{tabular}

\subsection{Zeros Pattern}

The zeros pattern of the z-transform in generating the sequence indicates a possible position of roots at the radius which the circle lies. As an alternative of having a good ACF properties, the zeros pattern can be edited by adding new zeros with the same angle in any length of the existing zero plot. Therefore, this procedure can be adopted to test the stability and for analyzing the systems by inserting the multiple methods.

Modulating pulse is achieved by transmitting the pulse that correlated the received signal. Radar, sonar and echography applications basically used in signal processing method by pulse compression is to increase the range solution and to reduce the noise [10].

This method is a procedure for obtaining the set of sequences by zeros plot movement using fdatools. The multilevel sequence also used as the initial sequence will be represented as zeros of the polynomial. This method is aiming to estimate the right place of the new coordinated accordance with the characteristic from the initial sequence. Figure 1 shows the flowchart for this procedure.

The probability of choosing the zero elements is different from the choosing a nonzero element. The sufficiently large field size whether the sequence is singular or not is determined by probability one by the zero pattern of the sequences, i.e. the zeros are located in zeros plot. To create the purely real multilevel sequence that have a poor ER or large ACF of side lobes, the plotting should lie either of two circles which one circle at radius $\mathrm{X}$ while the other radius at $\mathrm{X}-1$. In order to design the multilevel sequences, there are three types of problems that should be faced:

a) Firstly, choose the number of pulses in the sequence where the number of roots following by the algorithm varies the positions.

b) Secondly, the radius of one or the other circle must be chosen in the complex plane on which the root lies and the maximum values of ER are determined. This is to ensure that it increases exponentially with the number of root positions which have to be investigated.

c) Lastly, the roots must be decided on which circle that each root should lie.

The zeros plot on the z-plane are linear system. Matlab software used to generate the program function to view the pole-zero plot for this filter, it can be use as an example:

>>zplane (zer,pol)

To access to an additional tools, fvtool can be used. Firstly, the poles and zeros should be converted to the transfer function form and then the fvtool can be used.

$>>[\mathrm{b}, \mathrm{a}]=\mathrm{zp} 2 \mathrm{tf}$ (zer, pol, 1 ), where ' 1 ' is a gain

Designing Multilevel Sequences using $\mathrm{m}$ integer and $\mathrm{N}$ length are produced to obtain $\mathrm{m}(\mathrm{x})$ sequences. Table 1 shows the examples of some integer of Huffman sequences. It is generated by $\mathrm{m}$ integer and length of thirteen, the contents of that table also include the SLE, ER and PSL. Referring from that data, the best value of that parameter was found. Figure 2 shows zeros plot pattern movement for length $\mathrm{N}=13$ with the integer of $\mathrm{m}$ equivalent to one. The length of $\mathrm{N}$ should be an odd integer to generate $\frac{\mathrm{N}}{2}$. $\mathrm{N}$ lengths sequences are represented as zeros of the polynomials [11]. 


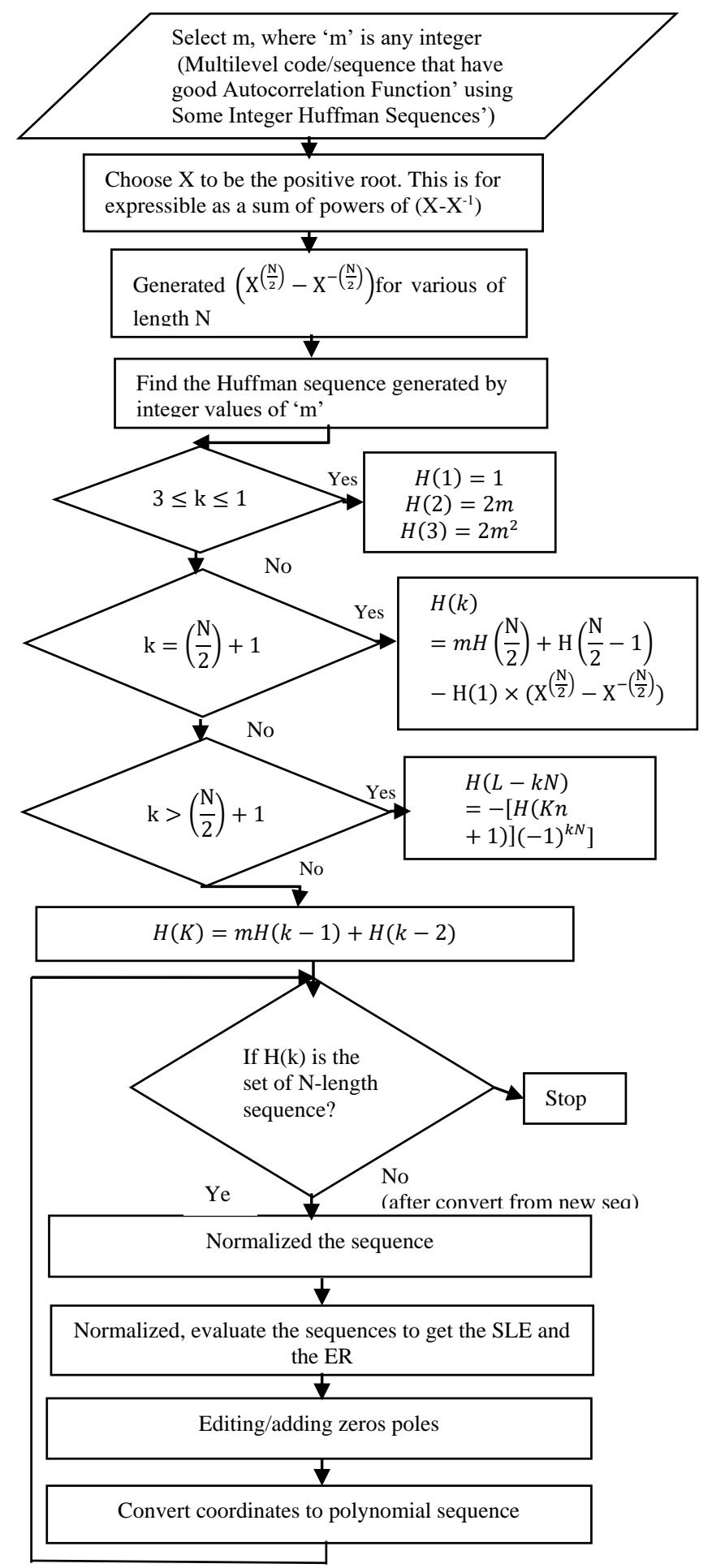

Figure 1. Flow chart of code plot coordinates with adding/editing zeros

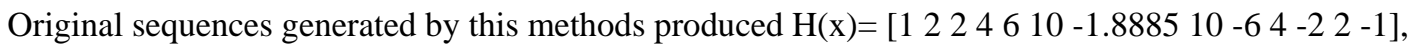
while SLE and ER is 1.1723 and 3.2557. 


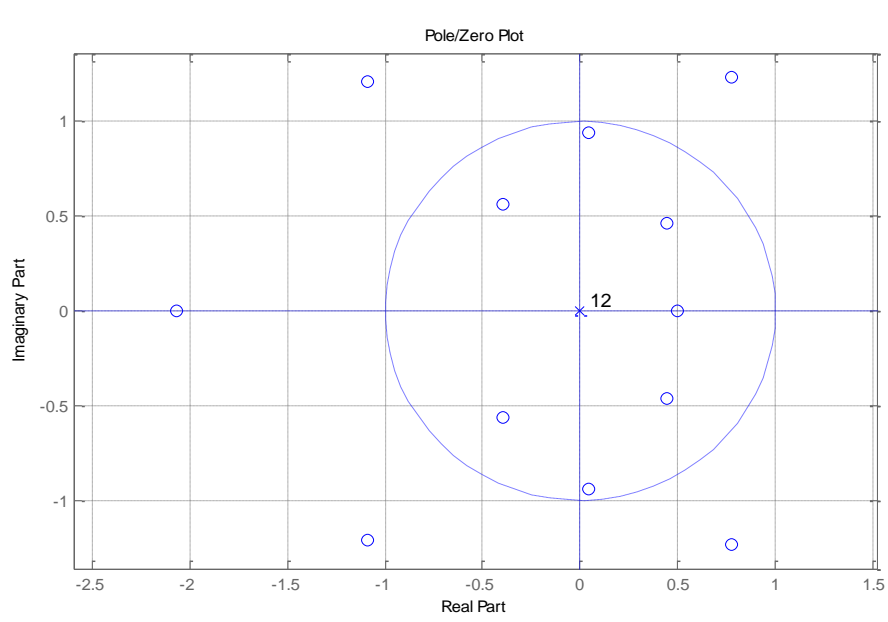

Figure 2. Zeros pattern for length-13 and integer $\mathrm{m}=1$

Using fdatools (Matlab software) to import filter from workspace to the numerator and denominator to enter that particular value at Pole Zero Editor. As a theory, when the z-plane of the zeros lies in the two balancing circle, the envelope of the pulse train tends to be relatively symmetrical in the mid-pulse [12]. All possible positions of the roots in the Z-domain are generated and each position which the radius circle lies has been optimized. The result of real and complex from the original sequences are presented in Table 2 . That table shows the rectangular and polar coordinates from original and editing pole defined to distinguish both results which is the best result discovered. Figure 3 shows the complex envelopes of the pulse train from the original and new editing from the thirteen zero patterns.

Table 2. Rectangular Coordinates and Polar Coordinates from Original and Editing/Adding Pole

\begin{tabular}{cccc}
\hline \multicolumn{2}{c}{ Rectangular Coordinates } & \multicolumn{2}{c}{ Rectangular Coordinates } \\
\hline \multicolumn{2}{c}{ Real } & Imaginary & \multicolumn{2}{c}{ Real } & Imaginary \\
Original pole & \multicolumn{2}{c}{ Editing/adding pole } \\
-2.068 & 0 & -1.0953 & 0 \\
-1.0854 & 1.2063 & -0.7553 & 0.8175 \\
-1.0854 & -1.2063 & -0.7553 & -0.8175 \\
0.7765 & 1.2366 & 0.5789 & 0.9090 \\
0.7765 & -1.2366 & 0.5789 & -0.9090 \\
0.0425 & 0.9428 & 0.04261 & 0.9875 \\
0.0425 & -0.9428 & 0.04261 & -0.9875 \\
-0.3955 & 0.5588 & -0.5198 & 0.7652 \\
-0.3955 & -0.5588 & -0.5198 & -0.7652 \\
0.4483 & 0.4642 & 0.6312 & 0.6605 \\
0.4483 & -0.4642 & 0.6312 & -0.6605 \\
0.4953 & 0 & 0.8928 & 0 \\
Polar Coordinates & Polar Coordinates \\
Magnitude & Angle & Magnitude & Angle (rad) \\
\multicolumn{4}{c}{} \\
Original pole & Editing/adding pole \\
2.068 & 3.1416 & 1.0953 & 3.1416 \\
1.6227 & 2.3035 & 1.113 & 2.3166 \\
1.6227 & -2.3035 & 1.113 & -2.3166 \\
1.4602 & 1.0101 & 1.0777 & 1.0038 \\
1.4602 & -1.0101 & 1.0777 & -1.0038 \\
0.9438 & 1.5257 & 0.9884 & 1.5277 \\
0.9438 & -1.5257 & 0.9884 & -1.5277 \\
0.6847 & 2.1867 & 0.9250 & 2.1675 \\
0.6847 & -2.1867 & 0.9250 & -2.1675 \\
0.6453 & 0.8029 & 0.9136 & 0.8081 \\
0.6453 & -0.8029 & 0.9136 & -0.8081 \\
0.4953 & 0 & 0.8928 & 0 \\
\hline \multicolumn{5}{c}{}
\end{tabular}




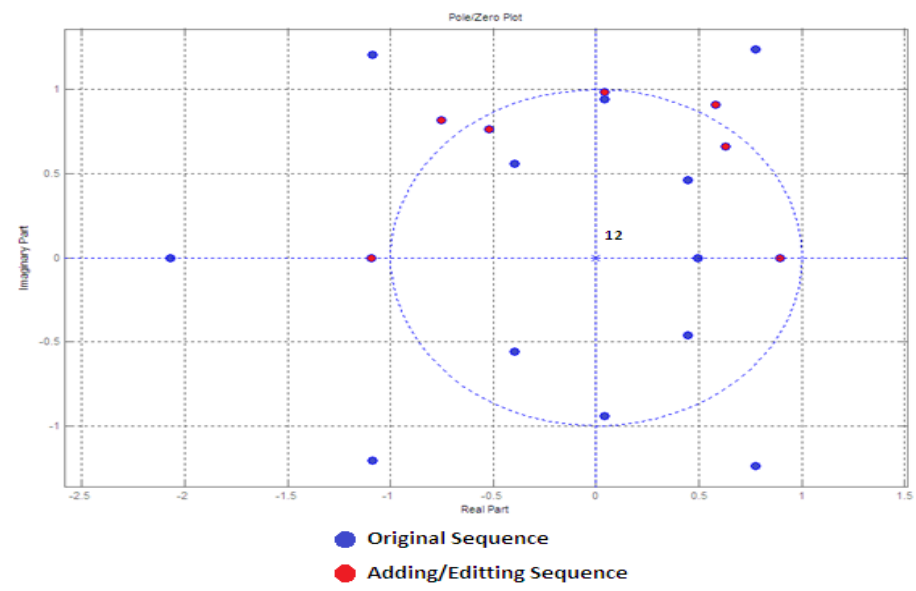

Figure 3. Complex Envelope of the Pulse Trains from the Original and editing Zeros pattern

\section{RESULTS AND DISCUSSION}

\subsection{Effect of Optimum Pulse Position}

In the original polynomials where the function of rectangular coordinates contains the zero-polegain such as poly (rec_coordinates) function. Figure 3 shows the movement of the root coordinates which tends to lie closer in the unit circle. That approached was done by implementing zeros plot (adding and editing) which has been proven effective in creating new illustrations. All of these zeros will be decided on their own about where the root in that circle should lie. Reference from two different sequences is a computational procedure which gives accurate result even when the SLE is low while the ER is mostly high. From the adding and editing method, real and imaginary axis from rectangular coordinates Table 1 illustrated as ' $p$ ' for evaluation by Matlab using the poly $(p)$ function where $p$ is a vector to return a row vector whose elements are the coefficients of the polynomial. With that, it produced the new set of sequence $\mathrm{H}(\mathrm{b})=$

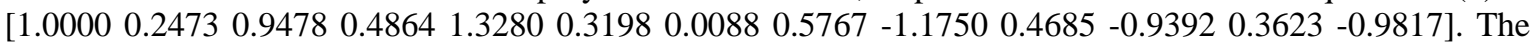
SLE of $\mathrm{H}$ (b) sequence is 1.4635 , while the ER is 4.5202 . Figure 4 and Figure 5 shows the autocorrelation function values of both sequences. Both of this graph have a positive result individually where the $\mathrm{Hx}$ sequences have the low SLE while the Hb have the best PSL and the details of the value is in Table 3 and

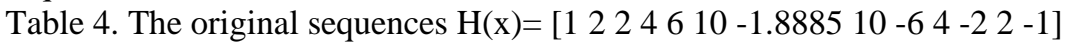

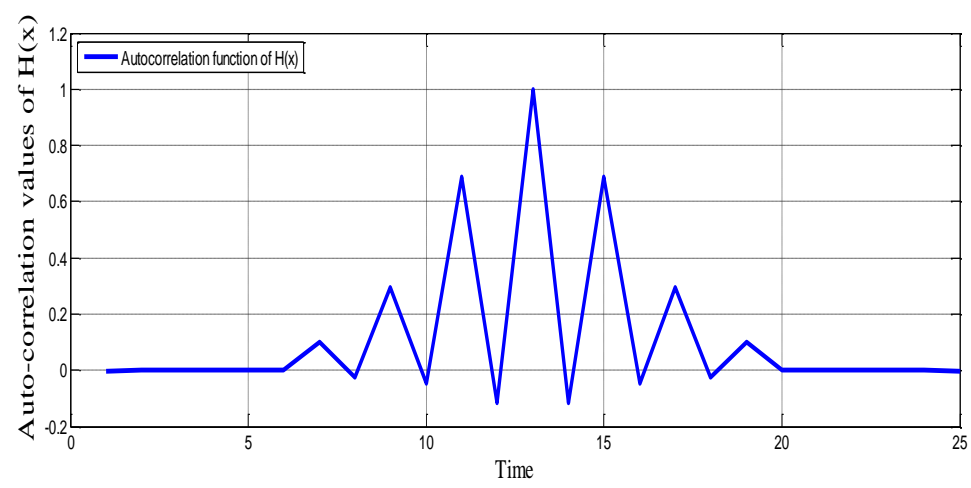

Figure 4. Autocorrelation Function of the Original Sequences (Hx)

Table 3. Correlation Values Obtained within input Sequences of Length $13(\mathrm{Hx})$

\begin{tabular}{|c|c|c|c|c|c|c|c|c|c|c|c|c|}
\hline$r_{0_{(h x)}}$ & $r_{1_{(h x)}}$ & $r_{2_{(h x)}}$ & $r_{3_{(h x)}}$ & $r_{4_{h x}}$ & $r_{5_{(h x)}}$ & $r_{6_{(h x)}}$ & $r_{7_{(h x)}}$ & $r_{8_{(h x)}}$ & $r_{9_{(h x)}}$ & $r_{10_{(h x)}}$ & $r_{11_{(h x)}}$ & $r_{12_{(h x)}}$ \\
\hline 1 & -0.1160 & 0.6880 & -0.0464 & 0.2949 & -0.0232 & 0.0983 & -0.0003 & 0 & -0.0024 & -0.0042 & -0.0049 & -0.0030 \\
\hline
\end{tabular}

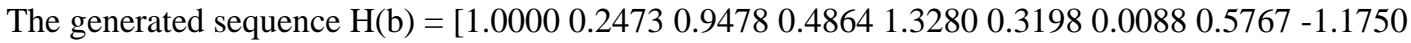
$0.4685-0.93920 .3623-0.9817]$ 


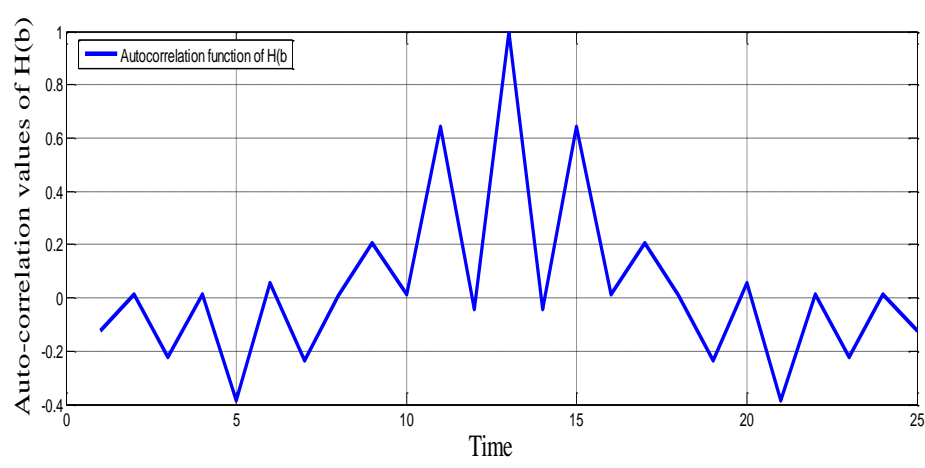

Figure 5. Autocorrelation Function of the Generated Sequences $(\mathrm{Hb})$

Table 4. Correlation Values Obtained within input Sequences of Length $13(\mathrm{Hb})$

\begin{tabular}{|c|c|c|c|c|c|c|c|c|c|c|c|c|}
\hline$r_{0_{(h b)}}$ & $r_{1_{(h b)}}$ & $r_{2_{(h b)}}$ & $r_{3_{(h b)}}$ & $r_{4_{h b)}}$ & $r_{5_{(h b)}}$ & $r_{6_{(h b)}}$ & $r_{7_{(h b)}}$ & $r_{8_{(h b)}}$ & $r_{9_{(h b)}}$ & $r_{10_{(h b)}}$ & $r_{11_{(h b)}}$ & $r_{12_{(h b)}}$ \\
\hline 1 & -0.0430 & 0.6440 & 0.0112 & 0.2057 & 0.0070 & $r_{6_{(h b)}}$ & $r_{7_{(h b)}}$ & $r_{8_{(h b)}}$ & $r_{9_{(h b)}}$ & $r_{10_{(h b)}}$ & $r_{11_{(h b)}}$ & -0.1231 \\
\hline
\end{tabular}

\subsection{Conditions for Convergence}

Convergence test is the method for testing the convergence of data collected prior to the previous one to get the roughly graph clearer. In Figure 6 shows the insignificant changing between the elements of two data even though it is known, that the Huffman code is the best sequence (13-length). Therefore, the multilevel sequence $\mathrm{H}(\mathrm{b})$ can be dignified as a good sequence. These sequences are:

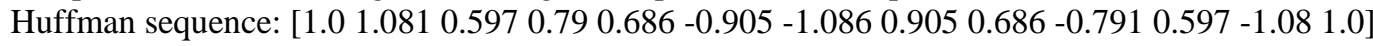

Multilevel sequence [generated sequence H(b)]: $\left[\begin{array}{llllll}1.0000 & 0.2473 & 0.9478 & 0.4864 & 1.3280 & 0.3198\end{array}\right.$ $0.00880 .5767-1.17500 .4685-0.93920 .3623-0.9817]$

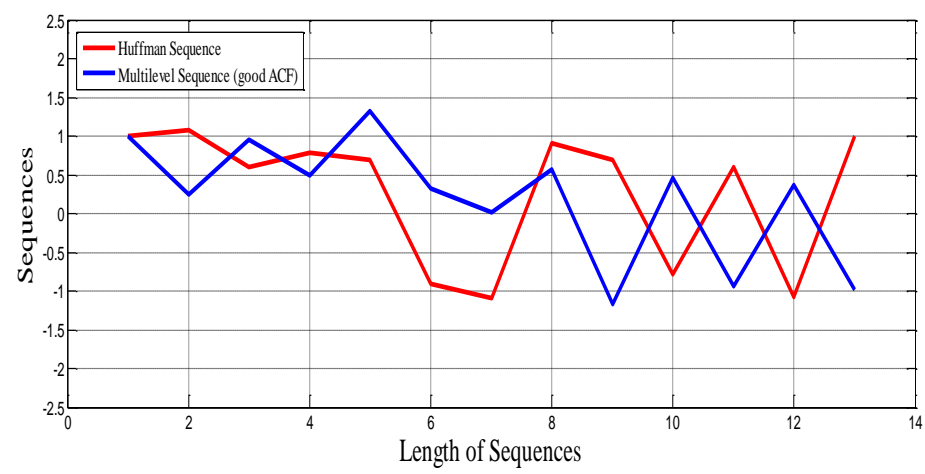

Figure 6. Graph plotting for Huffman Sequences and Multilevel Sequences that are good in the ACF

\section{CONCLUSION}

This work has been presented methods for the design of multilevel sequences that have pulse like autocorrelation functions and relatively high energy ratio. This accomplishes to investigations into discrete coding techniques for improving range resolution and clutter performance of radar systems. The waveform considered in this work besides representing an interesting mathematical area, are also of practical significance in related fields such as sonar, navigation, and digital communications.

The proposed methods contributes the generation of waveforms that have desirable properties for improving the range resolution of radar. Throughout this work digital processing has been assumed. The application of digital processing techniques to radar becomes more practical as compactness, cheapness and operational speed of digital micro circuits continue to increase. Although modern optical processing techniques sometimes provide an attractive alternative, the use of digital method with its inherent flexibility and reliability offers many advantages. To mention but a few, it simplifies pulse compression and real time multi-dimensional analysis of input data in range, Doppler, bearing, etc. Furthermore, it also offers 
considerable advantages in post detection and display processing. In addition the use of digital processor will in many cases reduce future system modifications to easy and inexpensive software changes, rather than requiring costly hardware replacements.

In conclusion, the multilevel code pulse trains design can be as the effective way to improve the overall performance of desired properties. It can enhance the radar performance by using the zeros pattern methods. It has the flexibility to dispose the noise ratio to improve the ER in that pulse trains. The ER valued is greater than original sequences but have poor characteristic of SLE compared between modified and without modification sequences but from the performance result it was efficient and flexible implementation compared the another methods, where it is the combination of 'inverse filtering' and 'clipping' provides a method of generating waveforms that have desirable properties for improving the range and Doppler resolution of radar [13-15].

\section{ACKNOWLEDGEMENTS}

This research was supported by UniMAP grant (STG 9001-00571). We are thankful to our colleagues who provided expertise that greatly assisted the research, although they not agree with all of the interpretations provided in this paper.

\section{REFERENCES}

[1] C. Jin et al., "Optimization of phase sequences design for TDCS-based multiple access system," 2016 8th International Conference on Wireless Communications \& Signal Processing (WCSP), Yangzhou, 2016, pp. 1-5.

[2] M. Soltanalian and P. Stoica, "Design of perfect phase-quantized sequences with low peak-to-average-power ratio," 2012 Proceedings of the 20th European Signal Processing Conference (EUSIPCO), Bucharest, 2012, pp. 25762580.

[3] X. Yi et al., "Phase Noise Effects on Phase-Modulated Coherent Optical OFDM," in IEEE Photonics Journal, vol. 8, no. 1, pp. 1-8, Feb. 2016.

[4] J. Xu, B. Bai, C. Dong, Y. Dong, Y. Zhu and G. Zhao, "Evaluations of Plasma Stealth Effectiveness Based on the Probability of Radar Detection," in IEEE Transactions on Plasma Science, vol. 45, no. 6, pp. 938-944, June 2017.

[5] A. M. Assem, R. M. Dansereau and F. M. Ahmed, "Adaptive sub-nyquist sampling based on haar wavelet and compressive sensing in pulsed radar," 2016 4th International Workshop on Compressed Sensing Theory and its Applications to Radar, Sonar and Remote Sensing (CoSeRa), Aachen, 2016, pp. 173-177.

[6] Y. Liu and P. H. Bauer, "On pole-zero patterns of non-negative impulse response discrete-time systems with complex poles and zeros," 2009 17th Mediterranean Conference on Control and Automation, Thessaloniki, 2009, pp. 1102-1107.

[7] N. N. Gorobets, V. I. Kiyko and V. N. Gorobets, "Analisys of cross-polarized radiation of optimized reflector antennas," 2013 International Kharkov Symposium on Physics and Engineering of Microwaves, Millimeter and Submillimeter Waves, Kharkiv, 2013, pp. 458-460.

[8] A. Polpetta and P. Banelli, "Design and performance of Huffman sequences in medical ultrasound coded excitation," in IEEE Transactions on Ultrasonics, Ferroelectrics, and Frequency Control, vol. 59, no. 4, pp. 630647, April 2012.

[9] MJ. M. Baden, M. S. Davis and L. Schmieder, "Efficient energy gradient calculations for binary and polyphase sequences," 2015 IEEE Radar Conference (RadarCon), Arlington, VA, 2015, pp. 0304-0309.

[10] S. Gupta, M. Zapf, H. Krauß and N. V. Ruiter, "Design of huffman sequences with limited bandwidth," 2014 IEEE International Ultrasonics Symposium, Chicago, IL, 2014, pp. 1089-1092.

[11] Y. Tanada and K. Sato, "Long huffman sequences derived from even functional quadratic residues," The Sixth International Workshop on Signal Design and Its Applications in Communications, Tokyo, 2013, pp. 56-59.

[12] S. Gupta, M. Zapf, H. Krauß and N. V. Ruiter, "Evaluation of Huffman Sequences based mismatched filter for bandwidth limited 3D USCT system," 2015 IEEE International Ultrasonics Symposium (IUS), Taipei, 2015, pp. 14.

[13] Ghani, F., Rosli, S.J., Ghani, A.H.A., Ahmad, B.R.: "Waveform Design for Improved Range and Doppler Resolution in Radar". In: Proceedings Third International Conference on Intelligent Systems, Modeling and Simulation ISMSI 2011, held at Kota Kina Balu, Malaysia, 8-10 February, 2012, pp 247-251.

[14] Rosli, S.J., Ghani, F., Ghani, A.H.A., and Ahmad, B.R.: "Synthesis of Finite Length Multi Level Sequences for Clutter Rejection in Radar". 2012 Fourth International Conference on Computational Intelligence, Communication Systems and Networks.

[15] Rosli, S.J., Ghani, F., Ghani, A.H.A., and Ahmad, B.R.: "An Itterative Technique of Amplitude and Phase Modulated Pulse Trains for Radars". Fourth International Conference on Control, Communication and Power Engineering, CCPE 2013, Bangalore. 


\section{BIOGRAPHIES OF AUTHORS}

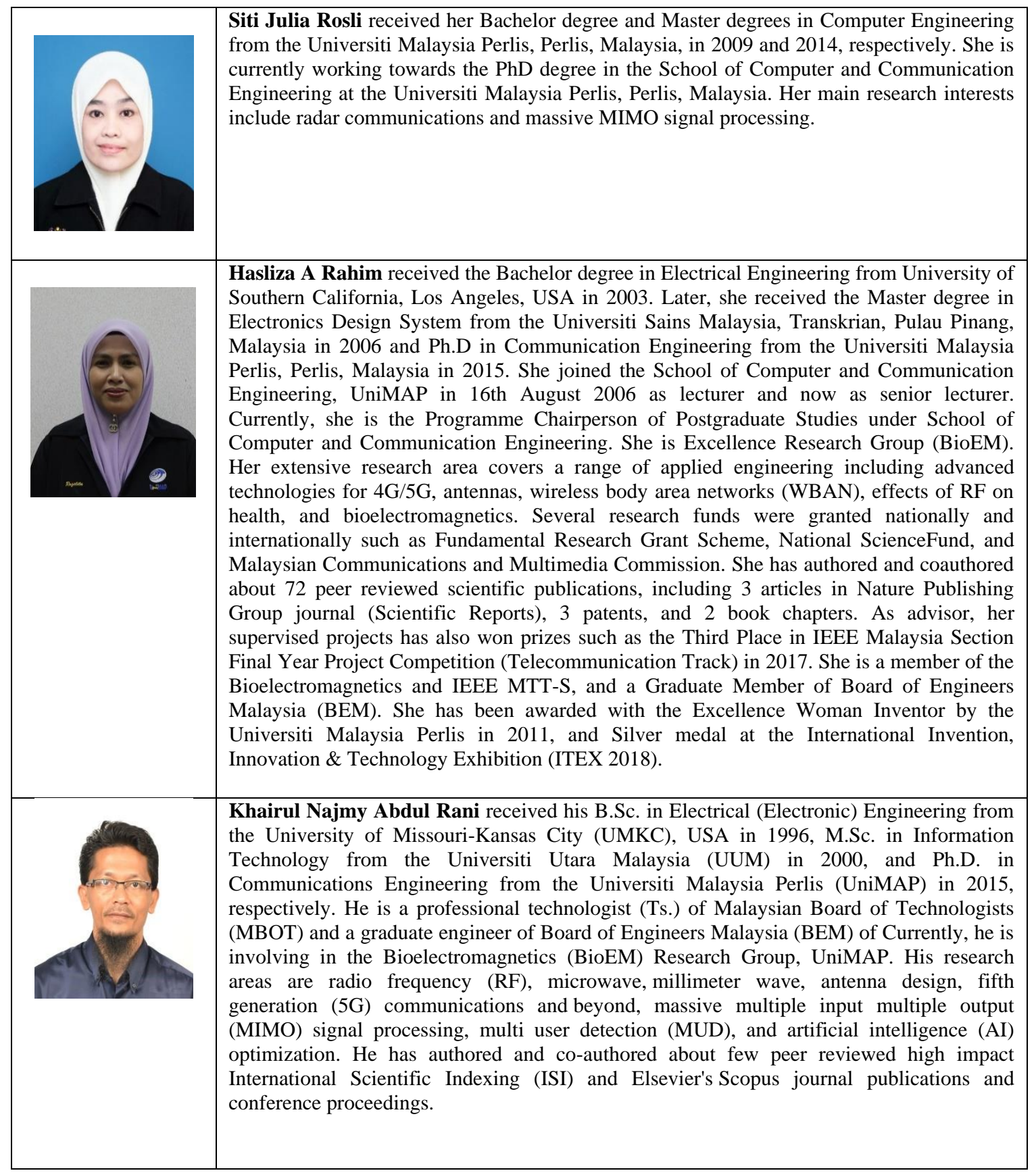

